\title{
Electrical Stimulation of the Suprahyoid Muscles in Brain-injured Patients with Dysphagia: A Pilot Study
}

\author{
Jaewon Beom, M.D., Sang Jun Kim, M.D., Tai Ryoon Han, M.D.
}

Department of Rehabilitation Medicine, Seoul National University College of Medicine, Seoul 110-744, Korea

\begin{abstract}
Objective To investigate the therapeutic effects of repetitive electrical stimulation of the suprahyoid muscles in brain-injured patients with dysphagia.

Method Twenty-eight brain-injured patients who showed reduced laryngeal elevation and supraglottic penetration or subglottic aspiration during a videofluoroscopic swallowing study (VFSS) were selected. The patients received either conventional dysphagia management (CDM) or CDM with repetitive electrical stimulation of the suprahyoid muscles (ESSM) for 4 weeks. The videofluoroscopic dysphagia scale (VDS) using the VFSS and American Speech-Language-Hearing Association National Outcome Measurement System (ASHA NOMS) swallowing scale (ASHA level) was used to determine swallowing function before and after treatment.

Results VDS scores decreased from 29.8 to 17.9 in the ESSM group, and from 29.2 to 16.6 in the CDM group. However, there was no significant difference between the groups ( $p=0.796)$. Six patients (85.7\%) in the ESSM group and 14 patients $(66.7 \%)$ in the CDM group showed improvement according to the ASHA level with no significant difference between the ESSM and CDM groups ( $\mathrm{p}=0.633$ ).

Conclusion Although repetitive neuromuscular electrical stimulation of the suprahyoid muscles did not further improve the swallowing function of dysphagia patients with reduced laryngeal elevation, more patients in the ESSM group showed improvement in the ASHA level than those in the CDM group. Further studies with concurrent controls and a larger sample group are required to fully establish the effects of repetitive neuromuscular electrical stimulation of the suprahyoid muscles in dysphagia patients.
\end{abstract}

Key Words Electrical stimulation, Brain, Dysphagia, Swallowing

\section{INTRODUCTION}

Dysphagia is a major complication known to develop in $37-78 \%$ of stroke patients ${ }^{1}$ which interferes with processes

Received September 3, 2009; Accepted December 27, 2010 Corresponding author: Tai Ryoon Han

Department of Rehabilitation Medicine, Seoul National University College of Medicine, 101, Daehak-ro, Jongno-gu, Seoul 110-744, Korea Tel: +82-2-2072-2925, Fax: +82-2-743-7473, E-mail: tairyoon@snu.ac.kr Copyright $\odot 2011$ by Korean Academy of Rehabilitation Medicine that transfer food material from the oral cavity to the stomach because of neurologic or structural disorders. Decreased laryngeal elevation due to weakened muscles involved in the swallowing process is a main cause of dysphagia in brain-injured patients, which can result in subglottic aspiration while swallowing.

Several methods have been established to treat dysphagia, and neuromuscular electrical stimulation (NMES) is one such method widely applied in the 
clinical setting. NMES of the pharyngeal area is known to strengthen the swallowing-related muscles by contraction in patients with dysphagia. Burnett et al. ${ }^{2}$ achieved laryngeal elevation in normal volunteers by stimulating the mylohyoid, geniohyoid, and thyrohyoid muscles using a hooked wire electrode. Leelamanit et al. $^{3}$ also found that synchronized surface electrical stimulation of the thyrohyoid muscle with swallowing has a therapeutic effect in dysphagia patients who did not improve with conventional treatment. Kim et al. ${ }^{4}$ applied electrical stimulation to the digastric and thyrohyoid muscles and found that functional dysphagia scale values between pre-treatment and 4 weeks following treatment were 13.20 (43.80-30.60) in the electrical stimulation group and $10.25(32.75-22.50)$ in the sham stimulation group.

However, other studies reported that pharyngeal electrical stimulation to the degree of contracting muscles lowers the hyoid bone by contracting the sternohyoid and omohyoid muscles, thereby interfering with the swallowing process. ${ }^{5,6}$ Humbert et al. ${ }^{6}$ reported that 'submental (suprahyoid) region-only' stimulation as well as stimulation of 'both submental and laryngeal (infrahyoid) regions simultaneously' at rest lowered the hyoid bone and larynx compared to the position before stimulation among healthy adults. However, paired stimulation of the submental region moved the hyoid bone upward compared to the stimulation of the laryngeal region. Kim and $\mathrm{Han}^{7}$ also reported that the degree of hyoid elevation during stimulation of the suprahyoid area was $66.8 \%$ greater than that during liquid swallowing among normal individuals. Based on these past studies, we aimed to evaluate the effect of repetitive NMES of the suprahyoid muscles for treating dysphagia in brain-injured patients with reduced laryngeal elevation.

\section{MATERIALS AND METHODS}

\section{Materials}

This study had a prospective, non-concurrent control comparative design. Among patients with dysphagia admitted to Seoul National University Hospital between January 2006 and July 2007, twenty-eight individuals (26 stroke and two traumatic brain injury patients) were enrolled who met the following criteria: (1) VFSS showing reduced laryngeal elevation during swallowing (judged by three rehabilitation physicians), (2) VFSS showing supraglottic penetration or subglottic aspiration when swallowing $2 \mathrm{ml}$ of $50 \%$ liquid barium (Solotop ${ }^{\circledR}$ suspension 140) solution, and (3) no clinical evidence of vagus or glossopharyngeal nerve palsy in addition to the inclusion criteria from the VitalStim ${ }^{\circledR}$ manual. $^{8}$

Only conventional dysphagia management (CDM) without electrical stimulation therapy was applied to patients admitted between January 2006 and March 2007 as a control group. Electrical stimulation of suprahyoid muscles (ESSM) was performed on patients between April 2007 and July 2007. Patients who received electrical stimulation also underwent CDM.

\section{Methods}

The protocol of this study was approved by the institutional review board of Seoul National University Hospital. The VDS ${ }^{9}$ and ASHA NOMS swallowing scale (ASHA level ${ }^{10}$ were used as parameters for evaluating swallowing function. The VDS (from 0 to 100) was determined using videofluoroscopic analysis, and consisted of several factors such as subglottic aspiration and food residues. This scale was reported to be sensitive and specific for detecting supraglottic penetration and subglottic aspiration, and was considered useful for predicting persistent dysphagia after stroke. ${ }^{11}$ The ASHA level is a measurement of both the supervision level required and diet level that intuitively reflects a patient's functional status. ${ }^{10}$

Treatments were performed for 30 minutes per day, 5 days per week for 4 weeks by three experienced occupational therapists who shared treatment protocols. Three rehabilitation physicians with considerable experience in the management of dysphagia (a senior professor, clinical fellow, and second-grade resident physician) determined the VDS and ASHA level before and after treatment with consensus. The VFSS was performed in a manner previously described ${ }^{9}$ and the VDS was determined while the patients swallowed $2 \mathrm{ml}$ of a $50 \%$ liquid barium solution.

Conventional dysphagia management consisted of ice cube training, double swallowing, effortful swallowing, Mendelsohn maneuver, and thermal tactile stimulation. If a patient could not swallow an ice cube without overt aspiration symptoms, ice cube training was excluded 
from CDM. All patients were sufficiently conscious to perform effortful swallowing and the Mendelsohn maneuver. STIMPLUS DP200 ${ }^{\circledR}$ (Cybermedic Corp., Iksan, South Korea) was used for suprahyoid NMES. The stimulation parameters used were $60 \mathrm{~Hz}$ of 500 microsecond duration with intermittent stimulation (1 second on and 1 second off) to avoid laryngeal spasms and to simulate normal pharyngeal swallowing. The active electrodes were placed at midpoints between the bilateral edges of the hyoid and chin, whereas the reference electrodes were placed at midpoints between the bilateral mandibular angle and chin (Fig. 1). The locations of active electrodes were at the points considered near to the motor point of the anterior belly of the digastric muscle. This was because we had previously shown that surface electrical stimulation moved the hyoid bone anteriorly and superiorly during stimulation using this electrode geometry in normal volunteers. ${ }^{7}$ Stimulus intensity was started at $3 \mathrm{~mA}$ and increased by $1 \mathrm{~mA}$. When a volunteer expressed discomfort but not pain, we administered the stimulus at this intensity three times to allow adaptation to the electrical stimulus. When a volunteer continued to complain about discomfort or pain, we defined the stimulus intensity used prior to the patient's complaint as the maximal tolerable intensity. We administered stimulation of the maximal tolerable intensity for 30 minutes using a 1 second on/1 second off cycle.

SPSS version 12.0 (Chicago, IL, USA) for Windows was

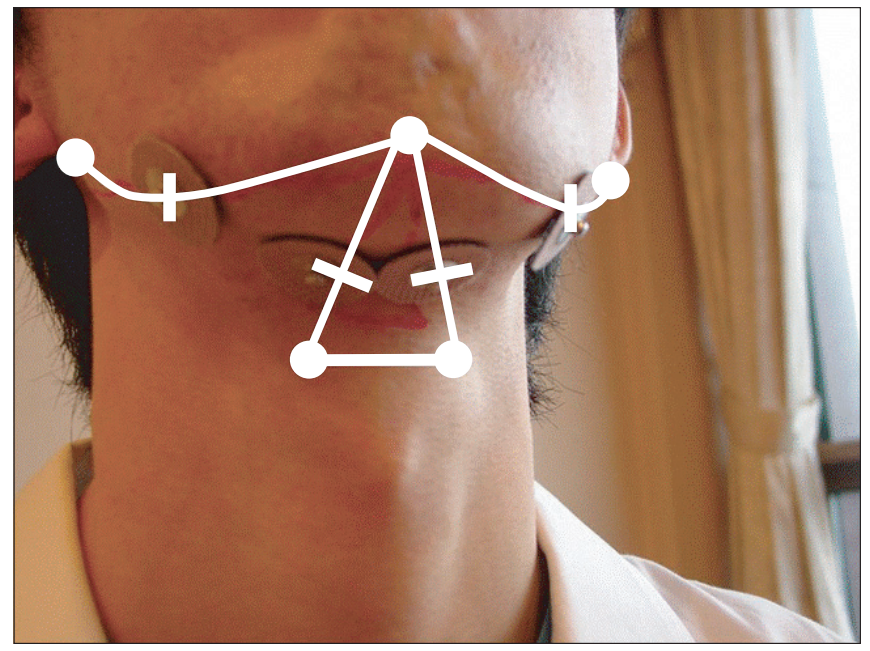

Fig. 1. Location of electrodes during electrical stimulation of the suprahyoid muscles. used for statistical analysis. The Mann-Whitney U test was used to confirm that initial swallowing function, patient age, and post-brain injury dysphagia onset were similar between the CDM and ESSM groups. To compare the degree of VDS score changes between the two groups, the Mann-Whitney U test was also used. Fisher's exact test was performed to compare the prevalence of ASHA level improvement in the CDM and ESSM groups. p-values less than 0.05 were considered statistically significant.

\section{RESULTS}

Seven patients were selected from the ESSM group and 21 from the CDM group. The average ages of patients were $66.1 \pm 19.5$ for the ESSM group and $68.5 \pm 12.5$ years for CDM group. The pre-treatment VDS was not significantly different $(\mathrm{p}=0.876)$ nor was the average patient age between the two groups $(\mathrm{p}=0.959)$. The time from brain injury to enrollment in this study was $2.4 \pm 2.1$ months for the ESSM groups, which tended to be longer than the 1.3 \pm 1.0 months for the CDM groups but was not statistically significant $(\mathrm{p}=0.249)$. Six patients in the ESSM group and nine in the CDM group had cortical lesions. One patient in the ESSM group and three in the CDM group had subcortical lesions, whereas all nine patients with brainstem lesion in our study were in the CDM group (Table 1). Maximal tolerable intensities varied

Table 1. General Demographics of the Patients in This Study

\begin{tabular}{|c|c|c|c|}
\hline & & ESSM group & CDM group \\
\hline Number & & 7 & 21 \\
\hline \multicolumn{4}{|l|}{ Gender } \\
\hline Male & & 3 & 9 \\
\hline Female & & 4 & 12 \\
\hline Age (years) & & $66.1 \pm 19.5$ & $68.5 \pm 12.5$ \\
\hline $\begin{array}{l}\text { Time since } \\
\text { brain injury } \\
\text { (months) }\end{array}$ & & $2.4 \pm 2.1$ & $1.3 \pm 1.0$ \\
\hline \multirow{3}{*}{$\begin{array}{l}\text { Location of } \\
\text { lesion (No.) }\end{array}$} & Cortex & 6 & 9 \\
\hline & Subcortex & 1 & 3 \\
\hline & Brainstem & 0 & 9 \\
\hline
\end{tabular}

ESSM: Electrical stimulation of suprahyoid muscles, CDM: Conventional dysphagia management 
from 5-12 mA in the ESSM group. Possible side effects of electrical stimulation therapy for dyaphagia including skin burns, pain, cardiovascular problems due to vagus nerve activation, and laryngeal spasm have been reported previously. ${ }^{12,13}$ In this study, however, no significant side effects were encountered other than transient pain which disappeared immediately after cessation of electrical stimulation.

\section{Videofluoroscopic dysphagia scale (VDS)}

The VDS score decreased from $29.8 \pm 13.5$ to $17.9 \pm 7.8$ in the ESSM group, and from $29.2 \pm 15.5$ to $16.6 \pm 11.8$ in the CDM group (Fig. 2). There was no significant difference between groups ( $\mathrm{p}=0.796$, Mann-Whitney $\mathrm{U}$ test). One patient in the ESSM group (patient No. 7) showed an increase in VDS score after treatment. This paradoxical VDS increase was caused by a newly developed premature bolus loss during liquid swallowing due to a dental prosthesis, and there were no findings such as motor weakness or dysarthria due to a new brain lesion.

\section{ASHA NOMS swallowing level}

Six patients $(85.7 \%)$ in the ESSM group and 14 patients (66.7\%) in the CDM group showed improved ASHA levels (Fig. 2), but there was no significant difference observed between the ESSM and CDM groups ( $p=0.633$, Fisher's exact test).

\section{DISCUSSION}

The use of NMES is becoming popular in clinical settings as several studies have been reported showing that pharyngeal NMES is effective for treating dysphagia in brain-injured patients. Carnaby-Mann and Crary ${ }^{14}$ performed a meta-analysis to examine the therapeutic effects of pharyngeal NMES on swallowing ability, and concluded that electrical stimulation tended to improve swallowing function. Freed et al. ${ }^{12}$ and Blumenfeld et
A

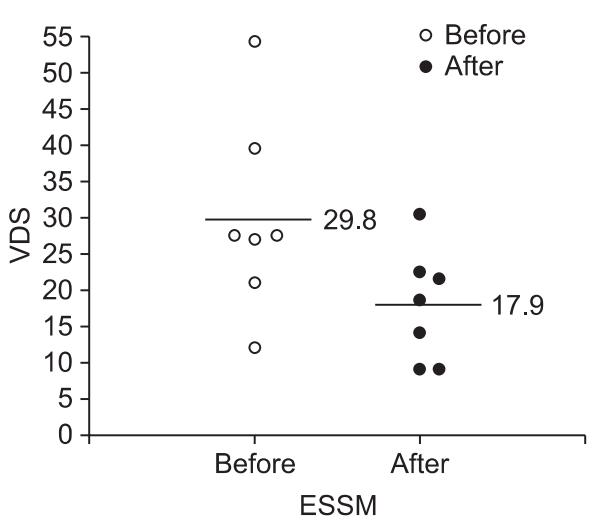

D

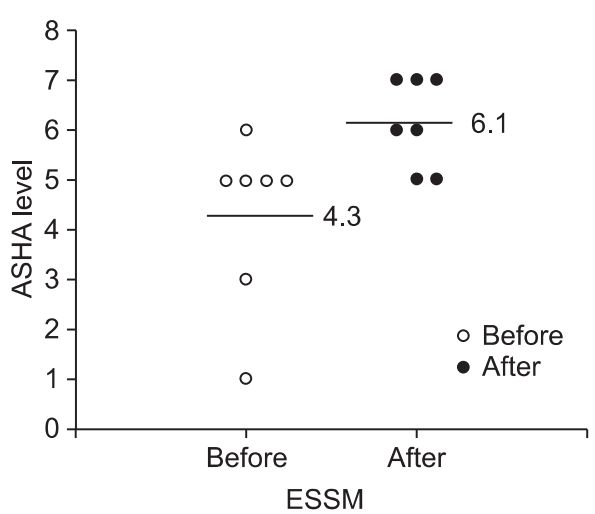

B

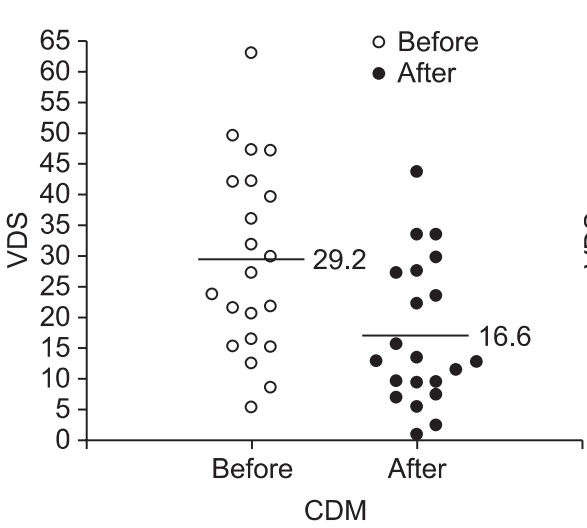

E

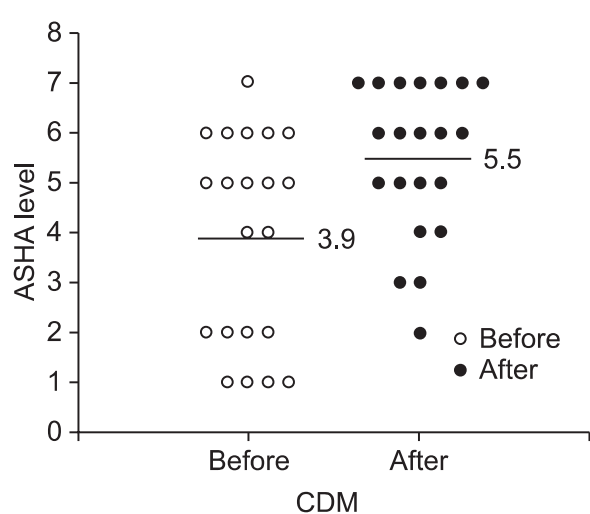

C

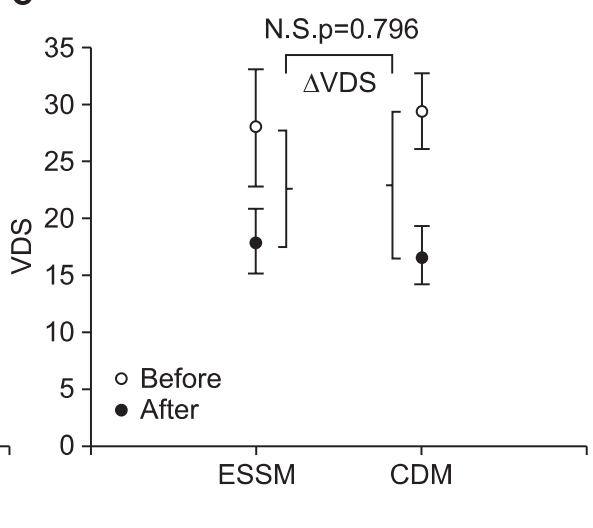

F

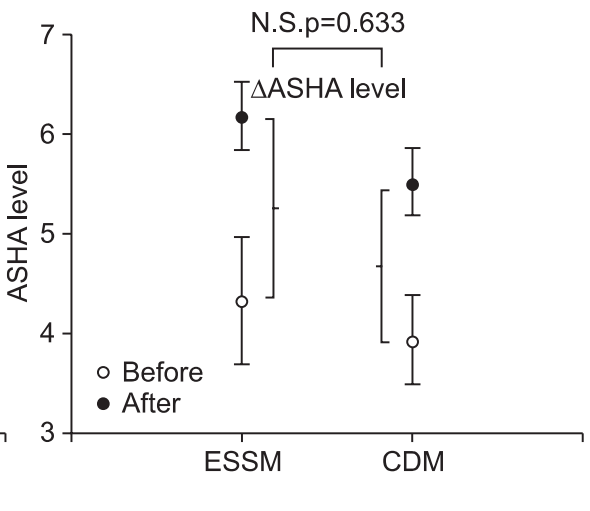

Fig. 2. VDS and ASHA levels in all patients before and after treatment. (A) VDS in the ESSM group. (B) VDS in the CDM group. (C) Comparison of $\triangle$ VDS between the ESSM and CDM groups. (D) ASHA level in the ESSM group. (E) ASHA level in the CDM group. (F) Comparison of $\triangle \mathrm{ASHA}$ level between the ESSM and CDM groups. N.S.: Not significant. 
al. ${ }^{15}$ reported that pharyngeal electrical stimulation is superior to CDM, and suggested that possible reasons were increase of local blood flow, reduced edema through decreased extracellular fluid, recruitment of more motor units than volitional contractions, selective activation of type II muscle fibers, and improvement of swallowing function by stimulating the sensory cortex of the cerebrum. ${ }^{15}$ Furthermore, Gow et al. ${ }^{16}$ found increased activities in the lateral cerebral cortex (Brodmann area 4,1 , and 2) after pharyngeal electrical stimulation in healthy volunteers which were confirmed by brain MRI and co-registered magnetoencephalography. Although pharyngeal NMES has been performed previously to strengthen swallowing-related muscles and reactivate neural pathways, the exact mechanism by which pharyngeal NMES improves dysphagia is not fully understood. Our study was designed to test whether the elevation of the hyoid bone improves swallowing function by applying electrical stimulation to suprahyoid muscles, whereas the above mentioned studies performed electrical stimulation of the thyrohyoid muscle.

The most likely reasons why repetitive NMES of the suprahyoid muscles in this study had no additive effect include the following. Surface electrical stimulation could be insufficient to strengthen the suprahyoid muscle although we observed anterior and superior displacement of hyoid bone by videofluoroscopy during the electrical stimulation. Suiter et al. ${ }^{17}$ found that stimulation at high frequencies such as $80 \mathrm{~Hz}$ can be inadequate for recruiting motor units involved in the swallowing process because the response of submental muscles to electrical stimulation is frequency-specific. Another study revealed that low frequency stimulation below $5 \mathrm{~Hz}$ is useful to treat dysphagia. ${ }^{18}$ Thus, the $60 \mathrm{~Hz}$ stimulation that we used in this study may not have been adequate. Since the ASHA level tended to increase more in the ESSM group than in the CDM group, electrical stimulation may be effective for improving swallowing function although there was no statistically significant difference. However, because this study included patients in the subacute phase (early stage of brain injury), spontaneous neurologic recovery might have masked the therapeutic effects. Although we selected the patients with similar post-onset periods of brain injury as a control group, limitations associated with adjusting for the effects of spontaneous neurologic recovery still remained.

When Type 1 error was assumed to be 0.05 , the power of this study was found to be low when determined using the PASS 2008 (NCSS, Utah, USA) because of the small sample size. Other limitations of this study included using a non-concurrent control group instead of a concurrent one, not checking suprahyoid muscle activity by electromyography during swallowing before or after treatment, and not comparing hyoid bone elevation and epiglottic rotation using a quantitative method by analyzing the videofluoroscopic results before or after treatment. Therefore, to explore the effect of repetitive NMES of the suprahyoid muscles on dysphagia more thoroughly, studies with a larger sample size and concurrent control group need to be conducted using quantitative methods.

\section{CONCLUSION}

Although repetitive NMES of the suprahyoid muscles did not further improve swallowing functions in patients with dysphagia and decreased laryngeal elevation, more patients had an improved ASHA level in the ESSM group than in the CDM group. Further studies with a larger sample size and concurrent control group are required to comprehensively establish the effects of repetitive NMES of the suprahyoid muscles on patients with dysphagia.

\section{REFERENCES}

1. Martino R, Foley N, Bhogal S, Diamant N, Speechley $M$, Teasell R. Dysphagia after stroke: incidence, diagnosis, and pulmonary complications. Stroke 2005; 36: 2756-2763

2. Burnett TA, Mann EA, Cornell SA, Ludlow CL. Laryngeal elevation achieved by neuromuscular stimulation at rest. J Appl Physiol 2003; 94: 128-134

3. Leelamanit V, Limsakul C, Geater A. Synchronized electrical stimulation in treating pharyngeal dysphagia. Laryngoscope 2002; 112: 2204-2210

4. Kim DY, Koh ES, Kang BS, Han TR, Lee SU. Effects of electrical stimulation for dysphagia caused by stroke. J Korean Acad Rehab Med 2008; 32: 9-14

5. Ludlow CL, Humbert I, Saxon K, Poletto C, Sonies B, Crujido L. Effects of surface electrical stimulation both at rest and during swallowing in chronic pharyngeal 
dysphagia. Dysphagia 2007; 22: 1-10

6. Humbert IA, Poletto CJ, Saxon KG, Kearney PR, Crujido L, Wright-Harp W, Payne J, Jeffries N, Sonies BC, Ludlow CL. The effect of surface electrical stimulation on hyolaryngeal movement in normal individuals at rest and during swallowing. J Appl Physiol 2006; 101: 1657-1663

7. Kim SJ, Han TR. Effect of surface electrical stimulation of suprahyoid muscles on hyolaryngeal movement. Neuromodulation 2009; 12: 134-140

8. Wijting Y, Freed M. VitalStim therapy training manual, 1st ed, Hixson: Chattanooga group, 2004, 108-109

9. Han TR, Paik NJ, Park JW. Quantifying swallowing function after stroke: a functional dysphagia scale based on videofluoroscopic studies. Arch Phys Med Rehabil 2001; 82: 677-682

10. Wesling M, Brady S, Jensen M, Nickell M, Statkus D, Escobar N. Dysphagia outcomes in patients with brain tumors undergoing inpatient rehabilitation. Dysphagia 2003; 18: 203-210

11. Han TR, Paik NJ, Park JW, Kwon BS. The prediction of persistent dysphagia beyond six months after stroke. Dysphagia 2008; 23: 59-64

12. Freed ML, Freed L, Chatburn RL, Christian M. Electrical stimulation for swallowing disorders caused by stroke. Respir Care 2001; 46: 466-474

13. Huckabee ML, Doeltgen S. Emerging modalities in dysphagia rehabilitation: neuromuscular electrical stimulation. N Z Med J 2007; 120: U2744

14. Carnaby-Mann GD, Crary MA. Examining the evidence on neuromuscular electrical stimulation for swallowing: a meta-analysis. Arch Otolaryngol Head Neck Surg 2007; 133: 564-571

15. Blumenfeld L, Hahn Y, Lepage A, Leonard R, Belafsky PC. Transcutaneous electrical stimulation versus traditional dysphagia therapy: a nonconcurrent cohort study. Otolaryngol Head Neck Surg 2006; 135: 754-757

16. Gow D, Hobson AR, Furlong P, Hamdy S. Characterising the central mechanisms of sensory modulation in human swallowing motor cortex. Clin Neurophysiol 2004; 115: 2382-2390

17. Suiter DM, Leder SB, Ruark JL. Effects of neuromuscular electrical stimulation on submental muscle activity. Dysphagia 2006; 21: 56-60

18. Power M, Fraser C, Hobson A, Rothwell JC, Mistry S, Nicholson DA, Thompson DG, Hamdy S. Changes in pharyngeal corticobulbar excitability and swallowing behavior after oral stimulation. Am J Physiol Gastrointest Liver Physiol 2004; 286; G45-50 